\title{
PowerEnergy2016-59167
}

\section{THERMODYNAMIC MODEL OF A SOLAR RECEIVER FOR SUPERHEATING OF SULFUR TRIOXIDE AND STEAM AT PILOT PLANT SCALE}

\author{
Alejandro Guerra Niehoff \\ German Aerospace Center \\ Cologne, Germany
}

\author{
Dennis Thomey \\ German Aerospace Center \\ Cologne, Germany
}

\author{
Moises A. Romero Gonzales \\ German Aerospace Center \\ Cologne, Germany
}

\author{
Martin Roeb \\ German Aerospace Center \\ Cologne, Germany
}

\author{
Hans-Peter Streber \\ German Aerospace Center \\ Cologne, Germany \\ Christian Sattler \\ German Aerospace Center \\ Cologne, Germany
}

\author{
Justin Lapp \\ German Aerospace Center \\ Cologne, Germany \\ Robert Pitz-Paal \\ German Aerospace Center \\ Cologne, Germany
}

\begin{abstract}
Within the European research project SOL2HY2, key components for a solar hybrid sulfur cycle are being developed and demonstrated at pilot scale in a real environment. Regarding the thermal portion, a plant for solar sulfuric acid decomposition is set up and initially operated at the research platform of the DLR Solar Tower in Jülich, Germany.

One major component is the directly irradiated volumetric receiver, superheating steam and $\mathrm{SO}_{3}$ coming from a tube-type evaporator to above $1000{ }^{\circ} \mathrm{C}$. At the design flow rate of sulfuric acid (50\%-wt.) of $11 / \mathrm{min}$, a nominal solar power of $57 \mathrm{~kW}$ is required at the receiver. With a flat ceramic absorber made from $\mathrm{SiC}$ and a flat quartz glass window, the design is based on lab scale reactors successfully demonstrated at the solar furnace of the German Aerospace Centre (DLR) in Cologne, Germany.

A flexible lumped thermodynamic tool representing the receiver, compiled to assess different configurations, is presented in detail. An additional raytracing model has been established to provide the irradiation boundaries and support the design of a conical secondary concentrator with an aperture diameter of $0.6 \mathrm{~m}$. A comparison with first experimental data (up to $65 \%$ nominal power), obtained during initial operation, indicates the models to be viable tools for design and operational forecast of such systems. With a provisional method to account for the efficiency of the secondary concentrator, measured fluid outlet temperatures (up to $1000{ }^{\circ} \mathrm{C}$ ) are predicted with deviations of $\pm 60{ }^{\circ} \mathrm{C}$. Respective absorber front
\end{abstract}

temperatures (up to $1200{ }^{\circ} \mathrm{C}$ ) are under-predicted by $100-200$ ${ }^{\circ} \mathrm{C}$, with lower deviations at higher mass flows. The measured window temperature (up to $700{ }^{\circ} \mathrm{C}$ ) mainly depends on the absorber front temperature level, which is well predicted by the model.

\section{INTRODUCTION}

The hybrid sulfur cycle (HyS) [1] is considered as a promising route for hydrogen production from renewable energy. It consists of two thermal steps to evaporate (1) and decompose (2) sulfuric acid to sulfur dioxide, and an electrochemical step (3) to convert water and sulfur dioxide to hydrogen and sulfuric acid, the latter being recirculated.

(1) $\mathrm{H}_{2} \mathrm{SO}_{4} \rightarrow \mathrm{H}_{2} \mathrm{O}+\mathrm{SO}_{3}$

$$
\begin{aligned}
\Delta H_{R} & =176 \frac{\mathrm{kJ}}{\mathrm{mol}} \\
\Delta H_{R} & =99 \frac{\mathrm{kJ}}{\mathrm{mol}} \\
\Delta H_{R} & =115 \frac{\mathrm{kJ}}{\mathrm{mol}} 1
\end{aligned}
$$$$
\text { (2) } \mathrm{SO}_{3} \rightarrow \mathrm{SO}_{2}+1 / 2 \mathrm{O}_{2}
$$$$
\text { (3) } 2 \mathrm{H}_{2} \mathrm{O}+\mathrm{SO}_{2} \rightarrow \mathrm{H}_{2} \mathrm{SO}_{4}+\mathrm{H}_{2}
$$

The theoretical reversible cell potential is approximately one-seventh compared to the conventional low temperature water electrolysis. Thus, the highest share of the required energy for water splitting is input as heat, which can be provided by concentrated solar power (CSP). This enables high thermal process efficiencies (potentially higher than $20 \%$

${ }^{1}$ Valid for an assumed operational cell potential of $600 \mathrm{mV}$, as realistic design target for the electrolyzer [2]. 
annual solar to hydrogen) and, according to process benchmarks as in KOLB et al. [3], the potential for competitive hydrogen production.

One key step of the solar HyS-cycle is to effectively couple solar thermal energy to steps (1) and (2), medium temperature evaporation $\left(<400{ }^{\circ} \mathrm{C}\right)$ and the high temperature decomposition $\left(>800{ }^{\circ} \mathrm{C}\right)$. Previous projects reported on directly irradiated concepts at laboratory scale, tested in the solar furnace in Cologne [4][5]. Evaporation and decomposition take place in two respective chambers with a directly irradiated porous ceramic structure (volumetric receiver), each closed by a quartz glass window. Due to limitations of kinetics and heat transfer in the depth of the honeycomb, the first few centimeters of the structure act as a solar receiver, heating up the incoming gases, while the remaining structure can virtually be considered an adiabatic reactor [6]. This led to an adapted design of the solar demonstration plant with separation of receiver and reactor in order to validate and later optimize both units independently from each other. The plant is being build up and operated at the Solar Tower in Jülich [7] within the European project SOL2HY2.

The plant has a capacity to process up to $11 /$ min of diluted sulfuric acid (50\%-wt.), corresponding to a heat requirement of the evaporator tubes of up to $60 \mathrm{KW}$. With irradiation and mass flux densities in the solar receiver of approximately $450 \mathrm{~kW} / \mathrm{m}^{2}$ and $0.18 \mathrm{~kg} / \mathrm{sec} \mathrm{m}^{2}$, respectively, this is the second known solar sulfuric acid decomposition reactor to be operated at relevant scale and under real environment. The last demonstration took place in 1985: a solar tube type reactor for sulfur trioxide decomposition, made from Hastelloy and arranged in an irradiated cavity, as investigated by General Atomics [8]. At that time, feasibility of this concept was proven for pressure operation up to 10 bar as well [9]. The conversion of $\mathrm{SO}_{3}$ was particularly limited by temperature and thus catalyst activity due to the available materials. Nevertheless, its principle may be of relevance for derived plant concepts with the application of improved materials $(\mathrm{SiC})$, e.g. for directly irradiated tubetype evaporation or even a bayonet-reactor arrangement [10].

A schematic of the new plant configuration is shown in Figure 2. An electrically powered evaporator provides steam and sulfur trioxide from liquid sulfuric acid. The gases are superheated to above $1000{ }^{\circ} \mathrm{C}$ in a closed volumetric receiver, which is the subject of the current work. $\mathrm{SO}_{3}$ will then be decomposed in an adiabatic reactor containing iron(III)-oxide $\left(\mathrm{Fe}_{2} \mathrm{O}_{3}\right)$ coated pellets, with expected performance similar as described in [11].

The design of the volumetric receiver required a flexible and easy to handle thermodynamic representation, in order to iteratively investigate geometric configurations, component sizes and operational parameters. For this purpose, a thermodynamic receiver model with lumped elements and a one-dimensional description of heat transfer within the absorber has been compiled and implemented in EES [12]. Irradiation boundary conditions have been determined with an additional raytracing tool considering the heliostat field, the tower, and the receiver geometry. The methodology and results of these models are presented and discussed in this paper. A complementary CFD analysis of the receiver has already been reported in [13] and a summary of the receiver design applying CAE tools has recently been presented in [14].

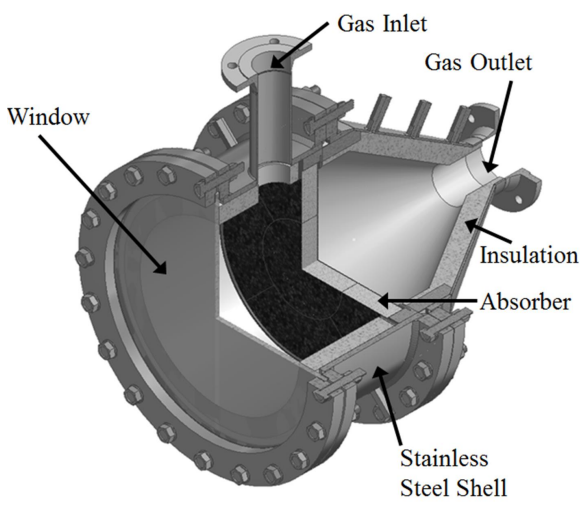

Figure 1: SOL2HY2 solar receiver for superheating of acidic gases

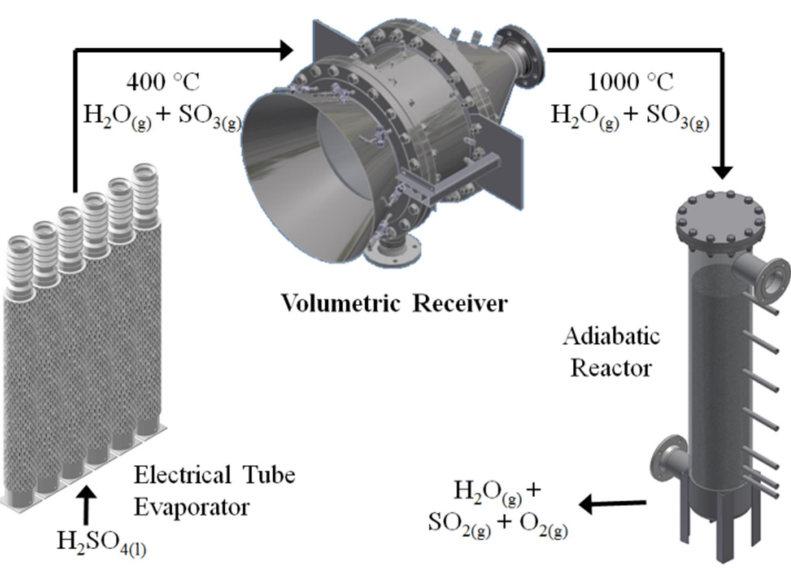

Figure 2: Plant set-up including the volumetric receiver with conical secondary concentrator

\section{NOMENCLATURE}

$\begin{array}{ll}\text { Abbreviations } & \\ \text { CAE } & \text { computer-aided engineering } \\ \text { CFD } & \text { computational fluid dynamics } \\ \text { CSP } & \text { concentrated solar power } \\ \text { DLL } & \text { dynamic link library } \\ \text { EES } & \text { Engineering Equation Solver } \\ \text { PDE } & \text { partial differential equation } \\ \text { ENEA } & \text { Italian National Agency for New } \\ & \text { Technologies, Energy and Sustainable } \\ & \text { Economic Development } \\ \text { DLR } & \text { German Aerospace Center } \\ \text { HycycleS } & \text { materials and components for hydrogen } \\ & \text { production by sulphur based thermochemical } \\ & \text { cycles } \\ \text { SOL2HY2 } & \text { solar to hydrogen hybrid cycles }\end{array}$

Abbreviations

CAE computer-aided engineering

CFD computational fluid dynamics

CSP

DLL

EES

PDE solar to hydrogen hybrid cycles 
Letters

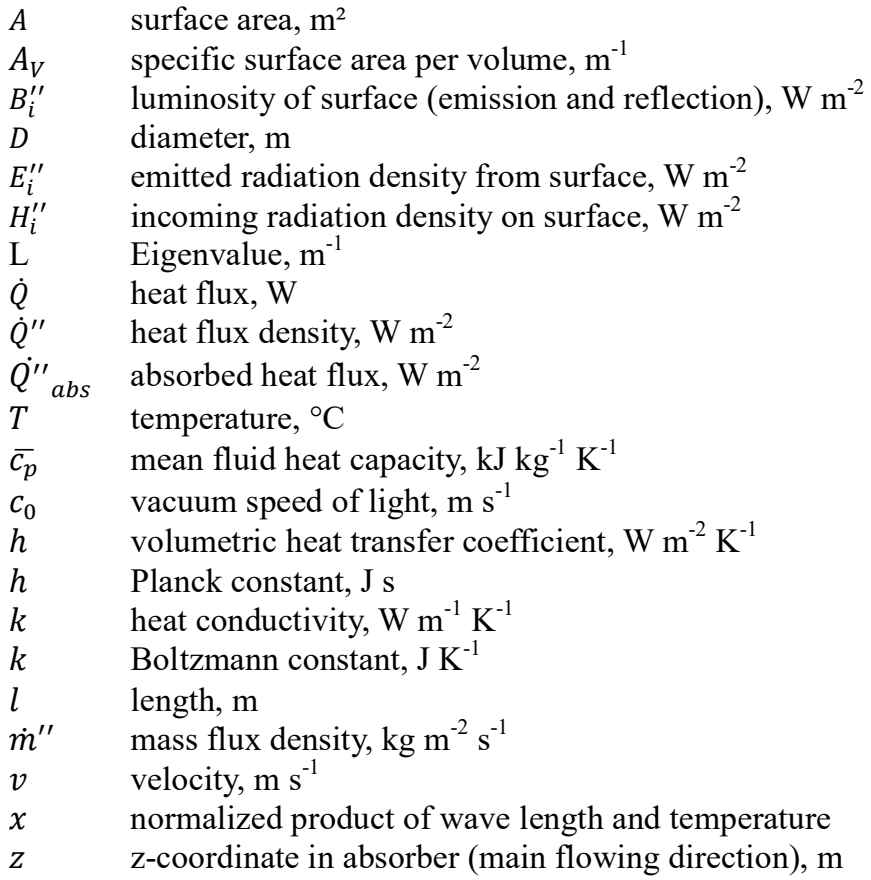

\section{Greek symbols}

$\Theta \quad$ temperature, $\mathrm{K}$

$\alpha \quad$ opening angle of secondary, ${ }^{\circ}$

$\epsilon_{i} \quad$ emissivity

$\epsilon \quad$ porosity or void fraction of the absorber material

$\eta \quad$ efficiency

$\lambda \quad$ wave length, $\mathrm{m}$

$\rho \quad$ density, $\mathrm{kg} \mathrm{m}^{-3}$

$\rho \quad$ reflectivity

$\tau \quad$ transmissivity

$\phi_{i, k} \quad$ viewing factor $i \quad$ considered (lumped) element (or surface)

abs / 1 absorber

cas / 2 casing

win / 3 window

digits indicate considered element surface for radiation heat exchange for better readability

$\infty \quad$ at infinite flow length

\section{THERMODYNAMIC RECEIVER MODEL}

The SOL2HY2 receiver is formed by a flat ceramic porous absorber in an insulated cylindrical or conical shaped casing, closed by a quartz glass window. The thermodynamic model considers radiation heat exchange between absorber, window, and insulation. One surface temperature determining radiation is assigned to each entity (lumped representation of each component). Energy balances for each entity furthermore determine heat transport (towards environment as losses or towards the fluid) and inner surface temperature. The implicit coupling to each other via the irradiation heat exchange requires an iterative solving of the system of equations. The model is implemented in EES [12] due to its powerful numerical solver and the availability of diverse species properties and thermodynamic correlation databases, required for the modelling.

All necessary modelling simplifications and assumptions are made in a conservative way (e.g. leading to an overestimation of material temperatures or underestimation of efficiency).

Transient and stationary solar receiver modelling is a standard design procedure to evaluate the potential of new receiver concepts. Feature of the stationary model described in the present contribution is the coupling of equations describing the heat transfer in a volumetric receiver with radiation heat exchange within a cavity closed by a quartz glass window, and the consideration of effective wave length dependent optical properties of the window. The lumped model is kept robust and might be further discretized by dividing the single element surfaces into zones to evaluate more sophisticated receiver shapes. Modelling of a similarly shaped closed conical cavity has been presented by PIATKOWSKI \& STEINFELD [2]. In contrast to the current contribution, the absorber is fully solid and heat is conductively transferred into another reaction cavity. This model however is further discretized and transient. For the development of the SOLREF-receiver, a closed cavity shaped receiver, the tool VORECO has been applied to assess the volumetric absorber surface temperature, discretized into several zones [4]. Further related modelling approaches can be found in [6] (volumetric flow receivers) and [8] (transient modelling of a volumetric receiver with reaction). 


\section{Optical Properties of the quartz glass window}

Quartz Glass features a high transmissivity of the solar spectrum $(>90 \%)$, good mechanical properties up to a diameter size of $600 \mathrm{~mm}$ high temperature resistance $\left(<800{ }^{\circ} \mathrm{C}\right)$, low thermal expansion $\left(<0.6 \times 10^{-6} \mathrm{~K}^{-1}\right)$ and thus thermal shock resistance, and good chemical stability. However, tensile stresses must be avoided. Quartz glass already proved to exhibit good performance in previous related projects [5], and is used for the SOL2HY receiver as well.

Crucial for the modelling is to consider effective absorption and transmission of irradiation from the solar field and from the receiver cavity (emitted by insulation and absorber). All wave length dependent coefficients are made available by the provider for an arbitrary window thickness, and shown in Figure 3 for $15 \mathrm{~mm}$.

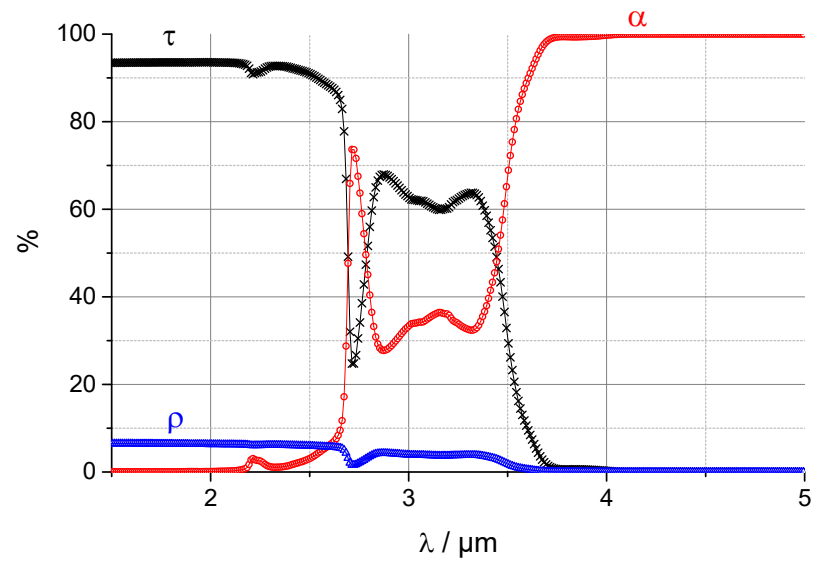

Figure 3: Optical properties of the quartz glass window for a thickness of $15 \mathrm{~mm}$ (data from provider). The transmission considers internal and external reflections.

The integral of Planck's normalized radiation function $F(0, x)$ universally describes the share of total emitted radiation for given body temperature and wavelength range [19]; $x$ being the normalized product of wave length and temperature:

$$
\begin{gathered}
x \equiv(\lambda T) \cdot \frac{k}{h c_{0}} \\
F(0, x)=\frac{15}{\pi^{4}} \int_{0}^{x} \frac{d x}{x^{5}\left(e^{1 / x}-1\right)}
\end{gathered}
$$

The total or effective transmission of radiation can thus be determined as function of the temperature of the emitting body $\mathrm{T}$ as follows (effective absorption and reflection analogous):

$$
\tau_{e f f(T)}=\int_{0}^{1} \tau_{(x)} \cdot d F(d x)
$$

The sum of all effective optical properties naturally remains unity. The procedure is applied to discrete temperature levels between $400{ }^{\circ} \mathrm{C}$ and $1400{ }^{\circ} \mathrm{C}$ as receiver component temperature range of interest. Resulting temperature dependent effective optical properties, drawn in Figure 4, can be regressed via a second order polygon with high precision. This method is thus easy to implement, robust and more precise then the usually applied 2- or 3-band approximation ${ }^{2}$. The wave dependent optical properties must be available or derived in adequate resolution and regressions must be re-determined for each investigated case (material, thickness).

Table 1 additionally summarizes discrete results for $5777 \mathrm{~K}$ and $1273 \mathrm{~K}$ as representative for the sun surface temperature and typical receiver temperature.

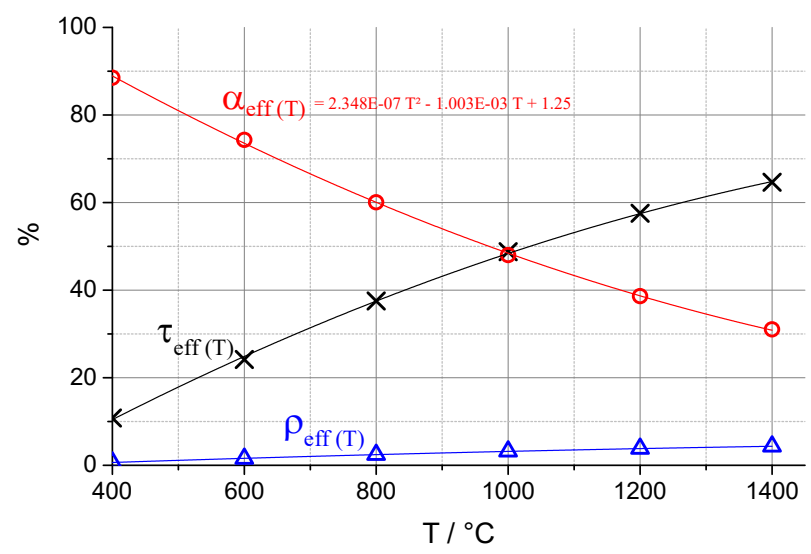

Figure 4: Resulting effective absorption, transmission, and reflection of radiation for quartz glass window as function of the emitting body temperature.

Table 1: Resulting effective absorption, transmission, and refection of radiation of quartz glass window arising from the sun or a 1000 ${ }^{\circ} \mathrm{C}$ hot black body.

\begin{tabular}{|l|c|c|}
\hline $\begin{array}{l}\text { Black body } \\
\text { temperature }\end{array}$ & $\begin{array}{c}5777 \mathrm{~K} \\
\text { (solar spectrum) }\end{array}$ & $\begin{array}{c}1273 \mathrm{~K} \\
\text { (representative for } \\
\text { receiver) }\end{array}$ \\
\hline Total transmission & $90.8 \%$ & $48.8 \%$ \\
\hline Total absorption & $2.6 \%$ & $48.0 \%$ \\
\hline Total reflection & $6.6 \%$ & $3.2 \%$ \\
\hline
\end{tabular}

\section{Global balances and re-radiation}

To determine the effective heat losses of the closed receiver, the geometry and re-radiation must be taken into consideration: radiation from the absorber will only partially leave the system; a considerable amount is reflected and absorbed by the insulation and window. Both in turn emit radiation into the cavity - partially facing the absorber.

${ }^{2}$ For an absorber front temperature of $1400{ }^{\circ} \mathrm{C}$, a 2-band approximation yielded window temperatures differing by more than $180{ }^{\circ} \mathrm{C}$, when the limit wave length was varied between 2.6 and $3.8 \mu \mathrm{m}$. 

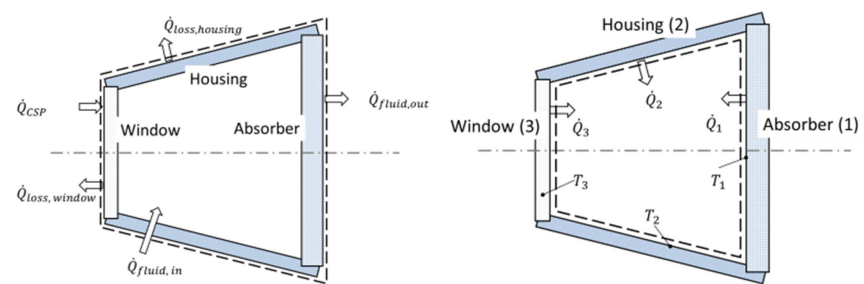

Figure 5: Lumped elements forming the receiver cavity. Left: overall energy balance. Right: energy balance within the cavity with re-radiation as exclusive heat transfer mechanism.

The overall energy balance describing the receiver system is depicted in Figure 5. At nominal operating conditions (absorber front temperatures higher than $1000{ }^{\circ} \mathrm{C}$ ) radiation is assumed to be the dominant mechanism to transfer heat from one element to another. Therefore, heat transport by conduction and convection within the cavity is neglected in the current approach. Heat streams and body temperatures are calculated iteratively by interconnected sub-models representing window, insulated casing, and absorber as lumped elements. Assuming grey and non-specular bodies, the enclosure method $[20,21]$ is applied to determine the effective radiative heat vectors as depicted in Figure 5:

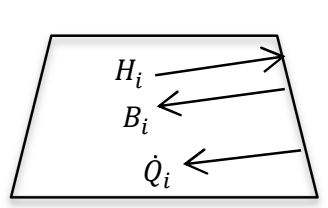

$$
\begin{aligned}
H_{i}^{\prime \prime} & =\sum_{k} B_{k}^{\prime \prime} \cdot \phi_{i, k} \\
B_{i}^{\prime \prime} & =E_{i}^{\prime \prime}+\sum_{k} \rho_{i} \cdot B_{k}^{\prime \prime} \cdot \phi_{i, k} \\
\dot{Q}_{i} & =\left(B_{i}^{\prime \prime}-H_{i}^{\prime \prime}\right) \cdot A_{i}
\end{aligned}
$$

Illustration and equations taken and adapted from [20]

Given the simplified geometry of the receiver (cylindrical or truncated cone), the corresponding viewing factors $\phi_{i, k}$ are dynamically determined in the code in order to enable varying diameter and interspace between absorber and window. The geometrical configuration is represented by two parallel circular surfaces with common middle axis. The corresponding viewing factors are determined according to [20].

All incoming concentrated solar power (CSP) heat is either absorbed by the process fluid or lost through the housing or the quartz glass window.

$$
\begin{aligned}
& \dot{Q}_{C S P}=\Delta \dot{Q}_{f}+\dot{Q}_{\text {loss }} \\
& \dot{Q}_{\text {loss }}=\dot{Q}_{\text {loss }, \text { win }}+\dot{Q}_{\text {loss }, \text { cas }} \\
& \Delta \dot{Q}_{f}=\dot{Q}_{f, \text { out }}-\dot{Q}_{f, \text { in }}=\dot{m} \cdot \overline{c_{p}} \cdot \Delta T_{f}
\end{aligned}
$$

Energy losses over the housing consist of convective and radiative heat. Energy losses over the window are the reflected amount of radiation as coming from the solar field, convective heat losses at the window surface, the radiation of the hot window towards environment, and the sum of transmitted radiation as determined by radiation exchange within the cavity.
Since no reaction is assumed to take place within the receiver, its thermal efficiency as central measure is defined as

$$
\eta_{t h}=\frac{\Delta \dot{Q}_{f}}{\dot{Q}_{C S P}}
$$

The sub-balances for the components are described in the following

\section{Heat loss of insulation and casing}

Heat transport through inner and outer insulation is considered straightforward via steady conduction through multiple layers in a cylindrical geometry in radial direction only. The metal casing is considered an ideal heat conductor. In case of conical shapes of the receiver, a mean outer and inner radius is used. The equations are coupled with the radiative and convective heat losses at the outer shell as well as with the reradiation balance in the cavity via the corresponding surface temperature.

\section{Energy balance of window}

The energy balance over the quartz glass window as shown in Figure 6 takes into account convective heat losses at the outside surface and radiation terms considering reflection transmission and absorption. Convective heat transfer at the inner side is neglected.

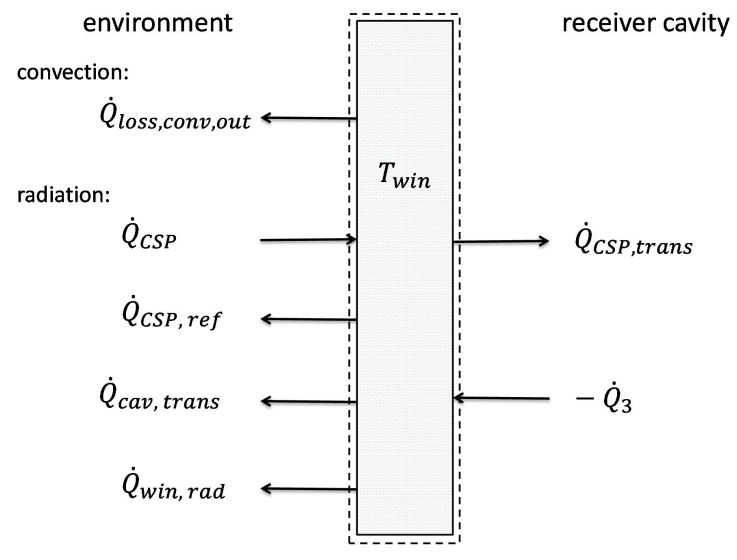

Figure 6: Energy balance over the lumped window entity

The coupling term to the energy balance in the inner cavity described by radiation heat exchange $\left(-\dot{Q}_{3}\right)$ already considers the reflected amount of irradiation coming from the cavity, as well as the emitted radiation into the cavity (see Figure 5). The net incoming heat flux is thus fully transmitted or absorbed. The summary of all heat lost from the window is

$$
\begin{gathered}
\dot{Q}_{\text {loss }, \text { win }}= \\
\dot{Q}_{C S P, \text { ref }}+\dot{Q}_{\text {win, rad }}+\dot{Q}_{\text {cav }, \text { trans }}+\dot{Q}_{\text {loss,conv }, \text { out }} \\
\text { and the energy balance on the window is } \\
\dot{Q}_{\text {loss,win }}+\dot{Q}_{C S P, \text { trans }}=\dot{Q}_{C S P}-\dot{Q}_{3}
\end{gathered}
$$


The window's temperature dependent heat terms depend on window surface area, and its temperature. Emissivity and the outer heat transfer coefficient are assumed as a fixed value. $\dot{Q}_{C S P, \text { ref }}$ and $\dot{Q}_{C S P, \text { trans }}$ are the net concentrated solar radiation as coming from the heliostat field reflected or transmitted through the window. The respective coefficients are found in Table 1, and absorbed solar power is considered implicitly.

The crux of the procedure is the determination of the radiation from the cavity transmitted through the window. Heat flux incoming from the cavity $H_{3}^{\prime \prime}$ consists of two shares assigned to absorber and insulation surface. Analogous, these terms are valued with the effective transmissivity $\tau_{\text {eff } i, k}$ as determined above (Figure 4):

$$
\begin{gathered}
H_{3}^{\prime \prime}=B_{1}^{\prime \prime} \cdot \phi_{3,1}+B_{2}^{\prime \prime} \cdot \phi_{3,2} \\
\dot{Q}_{\text {cav trans }}=\left(B_{1}^{\prime \prime} \cdot \phi_{3,1} \cdot \tau_{e f f 3,1}+B_{2}^{\prime \prime} \cdot \phi_{3,2} \cdot \tau_{e f f 3,2}\right) \cdot A_{3}
\end{gathered}
$$

\section{Energy balance of absorber}

The core of the one-dimensional description of heat transfer in the porous absorber is the analytical solution of following simplified coupled partial differential equation (PDE) system describing conservation of energy for the porous solid and the fluid:

$$
\begin{gathered}
0=h \cdot A_{V}\left(T_{f}-T_{s}\right)+k_{e f f} \frac{d^{2} T_{s}}{d z^{2}} \\
\dot{m}^{\prime \prime} \cdot \overline{c_{p}} \frac{d T_{f}}{d z}=h \cdot A_{v}\left(T_{s}-T_{f}\right)
\end{gathered}
$$

The term $h \cdot A_{V}$ can be interpreted as a volumetric heat transfer coefficient, which is determined via a ReynoldsNusselt correlation derived from experimental investigations of different materials and morphology applicable for volumetric receivers [22]. The equations are coupled through the heat transfer between the solid and the fluid.

In contrast to the solution of a similar PDE system by HOFFSCHMIDT [23], effective heat conductivity in the solid structure is considered here. Major physical simplifications still concern the heat source distribution due to penetrating radiation and heat transport mechanisms in the solid material. The effective heat conductivity in the material is approximated by scaling with porosity: $k_{e f f}=k_{b}(1-\epsilon)$. Heat transport by reradiation could be considered by adapting $k_{e f f}$ in future works. The conductivity of the fluid is of much lower order compared to heat transport by convection.

The solution is based on the transformation into three PDEs of first order by the introduction of an auxiliary variable $\gamma=\frac{\partial T_{w}}{\partial z}$, the determination of the corresponding characteristic polynomial and its three Eigenvalues [24]. Three boundary conditions concerning the known fluid inlet temperature at absorber inlet, the solid temperature gradient at $\mathrm{z}=0$, and thermal equilibrium for infinite flow length are applied to solve the resulting equation system:

$$
\begin{gathered}
\left|T_{f}\right|_{z=0}=T_{f, 0} \\
\left|\frac{d T_{s}}{d z}\right|_{z=0}=\frac{\dot{Q^{\prime \prime}}}{k_{\text {eff }}} \\
\left|T_{s}\right|_{z \rightarrow \infty}=\left|T_{f}\right|_{z \rightarrow \infty}
\end{gathered}
$$

The resulting analytical solution describes the fluid outlet temperature and absorber temperature for given absorbed power, mass flux density and fluid inlet temperature as function of the flow length:

$$
\begin{gathered}
T_{f}=T_{f, 0}+\frac{\dot{Q^{\prime \prime}}{ }_{a b s}}{\dot{m}^{\prime \prime} \cdot \overline{\overline{c_{p}}}} \cdot\left(1-e^{L \cdot z}\right) \\
T_{s}=T_{f, \infty}-\frac{\dot{Q^{\prime \prime}}{ }_{a b s}}{k_{e f f}} \cdot \frac{1}{L} \cdot e^{L \cdot z}
\end{gathered}
$$

$\mathrm{L}$ is the relevant Eigenvalue of the characteristic polynomial (equal or lower than zero), its unit being $\mathrm{m}^{-1}$ :

$$
L=-\frac{1}{2} \widehat{B}-\sqrt{\left(\frac{1}{2} \widehat{B}\right)^{2}+\widehat{A}}
$$

$\widehat{A}$ describes the ratio of the ability to transfer and conduct heat in the solid; $\widehat{A}=\frac{h \cdot A_{V}}{\mathrm{k}_{\mathrm{eff}}} . \widehat{B}$ represents the ratio of the ability to transfer heat to the fluid and its ability to absorb it; $\widehat{\mathrm{B}}=\frac{\mathrm{h} \cdot \mathrm{AV}_{\mathrm{V}}}{\dot{m}^{\prime \prime} \cdot \overline{c_{p}}}$.

The resulting temperature course for a nominal operational case is shown in Figure 7 with a net absorbed heat of approximately $25 \mathrm{~kW}$ and other parameters as given in Table 2. The volumetric heat transfer coefficient varies between 25 and $50 \mathrm{~kW} \mathrm{~K}^{-1} \mathrm{~m}^{-3}$ between absorber inlet and outlet due to the increasing velocity while the fluid is heated up within the absorber and the temperature dependent properties. Two principal methods to consider the $\mathrm{z}$-dependence of the heat transfer coefficient are conceivable: (1) a constant value is applied, (a) for conditions at the absorber front, (b) for conditions at the absorber outlet, (c) a mean value, or (2) the value for each position is applied as representative for the current run length. Approach (1a, broken lines) yields a longer run length to reach thermal equilibrium. Approaches ( $1 \mathrm{~b}$ and 2 , dotted and continuous lines) deliver quite close results and are considered as most reasonable. For this investigated case, the approaches do have a negligible impact on absorber front and fluid outlet temperature, and thus on the overall receiver model, if the absorber thickness is appropriately chosen to ensure thermal equilibrium at the outlet. 


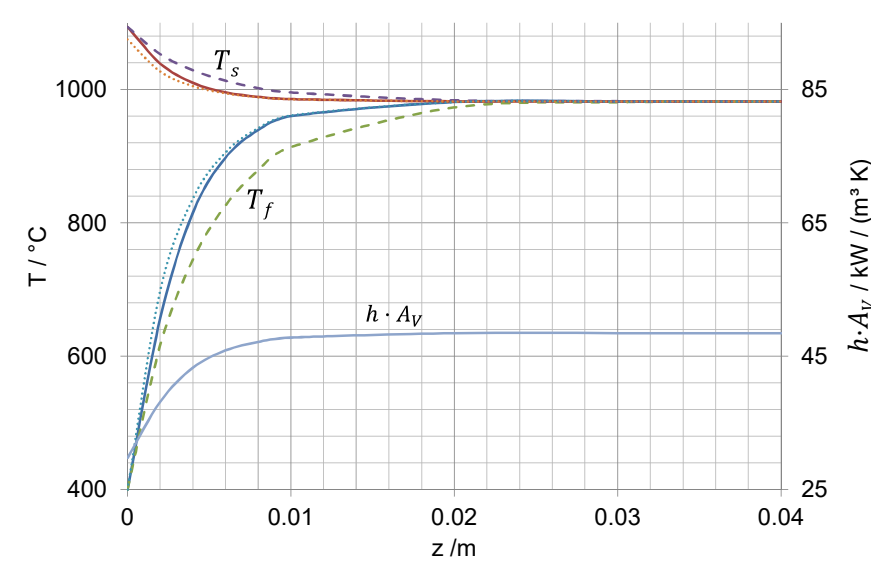

Figure 7: Resulting temperature course of solid and fluid in the absorber. Three approaches to consider the z-dependent heat transfer coefficient are shown.

Naturally, this system can be applied to swap any free or fixed variable, or to rapidly estimate fluid outlet temperature (assuming that thermal equilibrium is reached at the outlet of the absorber; $\rightarrow \infty)$ or absorber front temperature $(z=0)$. The fluid outlet temperature is then plausibly reduced to a simple overall energy balance, and the solid temperature at the front depends on the ability of the material to transfer heat into the depth and to fluid.

$$
\begin{gathered}
T_{f, \infty}=T_{f, 0}+\frac{\dot{Q^{\prime \prime}} a b s}{\dot{m}^{\prime \prime} \cdot \overline{c_{p}}} \\
T_{s, 0}=T_{f, \infty}-\frac{\dot{Q}_{a b s}^{\prime \prime}}{k_{e f f}} \cdot \frac{1}{L}
\end{gathered}
$$

The model does not account for the penetration of radiation into the depth of the porous structure. Due to the mathematical assumption that all heat is absorbed at $\mathrm{z}=0$, the front wall temperature is overestimated and the fluid outlet temperature correspondingly underestimated; which is compatible to the requirement of conservative design (v.s.).

Simplified measures for the thermal efficiency of the absorber, described by fluid and solid properties only, can be derived from these equations, e.g. the ratio between the rise in fluid temperature and the difference between fluid outlet and absorber front temperature.

\section{Irradiation}

The terms $H_{i}^{\prime \prime}$ describing the radiation heat exchange are extended to couple to the CSP heat transmitted through the window, powering absorber and the inner insulation. The transmitted CSP radiation partially hits absorber (1) and inner insulation (2), resulting in:

$$
H_{1}^{\prime \prime}=\sum_{k} B_{k}^{\prime \prime} \cdot \phi_{1, k}+\dot{Q}_{C S P, \text { trans }} \cdot \frac{\delta_{C S P}}{A_{1}}
$$

$$
H_{2}^{\prime \prime}=\sum_{k} B_{k}^{\prime \prime} \cdot \phi_{2, k}+\dot{Q}_{C S P, \text { trans }} \cdot \frac{\left(1-\delta_{C S P}\right)}{A_{2}}
$$

$\delta_{C S P}$ gives the fraction of solar energy transmitted through the window that is incident on the absorber, while the remainder is incident on the receiver walls:

$$
\begin{aligned}
\dot{Q}_{C S P, \text { trans }} & =\dot{Q}_{C S P, 1}+\dot{Q}_{C S P, 2} \\
\delta_{C S P} & =\frac{\dot{Q}_{C S P, 1}}{\dot{Q}_{C S P, \text { trans }}}
\end{aligned}
$$

The factor was determined from raytracing calculations described below, and is naturally influenced by receiver geometry, amount of active heliostats, sun position (date, time), and other factors.

\section{Studies with receiver model and results}

Operational and design parameters can freely be varied in order to investigate different configurations and cases. The impact of geometry and tuning factors (heat transfer coefficients, incident radiation on absorber) on material temperatures and efficiency was iteratively analysed during the design phase. Parameter variations yielded that for the chosen absorber morphology and properties (siliconized silicon carbide (SiSiC), 20 ppi, approximately $80 \%$ porosity), an absorber thickness of $0.04 \mathrm{~m}$ guarantees thermal equilibrium between fluid and solid, even at the most extreme expected conditions and worst case approaches. The final set for the nominal operation with $1 \mathrm{l} / \mathrm{min}$ of sulfuric acid is summarized in Table 2.

Table 2: Final geometry and nominal operating conditions of the receiver after iterative parameter studies.

\begin{tabular}{|l|l|l|}
\hline Parameter & Symbol & Value \\
\hline Absorber Diameter & $D_{\text {abs }}$ & $40 \mathrm{~cm}$ \\
\hline Distance from Window to Absorber & $L_{\text {cav }}$ & $20 \mathrm{~cm}$ \\
\hline Absorber Thickness & $L_{\text {abs }}$ & $4 \mathrm{~cm}$ \\
\hline Insulation Thickness & $L_{\text {ins }}$ & $6 \mathrm{~cm}$ \\
\hline Gas Inlet Temperature & $T_{\mathrm{f}, 0}$ & $400{ }^{\circ} \mathrm{C}$ \\
\hline Sulfuric Acid Flow Rate & $\dot{V}_{\mathrm{SA}, 0}$ & $11 \mathrm{~min}^{-1}$ \\
\hline Aperture Radiative Flux & $q_{\mathrm{CSP}}^{\prime \prime}$ & $450 \mathrm{~kW} \mathrm{~m}^{-2}$ \\
\hline Fraction of Radiation on Absorber & $\delta_{\mathrm{CSP}}$ & 0.7 \\
\hline Absorber Emissivity & $\varepsilon_{\mathrm{abs}}$ & 0.99 \\
\hline Insulation Thermal Conductivity & $k_{\text {ins }}$ & $0.31 \mathrm{~W} \mathrm{~m}^{-1} \mathrm{~K}^{-1}$ \\
\hline Shell Emissivity & $\varepsilon_{\text {shell }}$ & 0.7 \\
\hline Window Heat Transfer Coefficient & $h_{\text {window }}$ & $50 \mathrm{~W} \mathrm{~m}^{-2} \mathrm{~K}^{-1}$ \\
\hline
\end{tabular}

Variation of the diameter while keeping a cylindrical shape has already been shown and discussed in [13]: a diameter of $0.4 \mathrm{~m}$ provided a good trade-off between expected homogeneity of mass and irradiation flux, thermal efficiency, and maximum material temperatures. An alternative variation of window and absorber diameter, implying open or closed cavity shapes, 
naturally showed a more pronounced impact on temperatures and receiver efficiency. However, the option to increase the thermal efficiency by a closed geometry was rejected, since the cylindrical shape features better mountability and a higher degree of flux homogeneity. This configuration also exhibits a satisfactory robustness against changes in the fraction of solar irradiation incident the absorber $\delta_{C S P}$ [13].

An even mass flux distribution was confirmed by CFD methods with a cavity length of $0.2 \mathrm{~m}$ [13]. With this geometric configuration and at nominal conditions, outer heat transfer coefficients above $50 \mathrm{~W} / \mathrm{m}^{2} \mathrm{~K}$ yielded window temperatures below $800{ }^{\circ} \mathrm{C}$, which is a conservative design limitation for fused silica [25]. This corresponds to moderate forced convection, which is realized via 12 air nozzles circumferentially placed between window flange and secondary concentrator in the final design of the system.

Given the final parameter set as in Table 2, an operational base line receiver characteristic to provide an outlet temperature of $1000{ }^{\circ} \mathrm{C}$ is shown in Figure 8. The required incident solar power and resulting material temperatures and thermal efficiency is given as function of the sulfuric acid mass flow. The nominal operating conditions of $1 \mathrm{l} / \mathrm{min}$ and $57 \mathrm{~kW}$ on aperture (mass and irradiation flux density of $0.18 \mathrm{~kg} \mathrm{~m}^{-2} \mathrm{~s}$ and $450 \mathrm{~kW} \mathrm{~m}^{-2}$, respectively) are an order of magnitude higher compared to the preceding project HycycleS [5], and close to conditions for industrial operation [6].

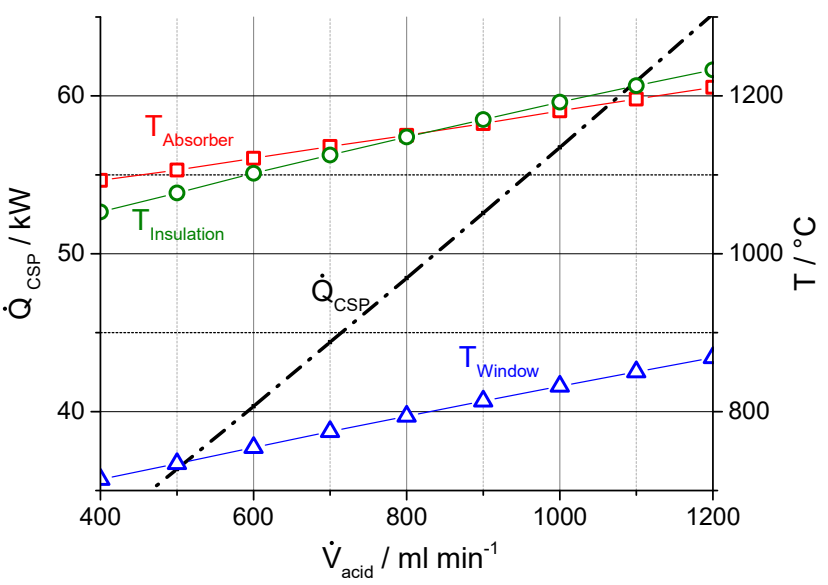

Figure 8: Operational characteristic of required incident CSP power on window and material temperature to raise the fluid temperature from $400{ }^{\circ} \mathrm{C}$ to $1000^{\circ} \mathrm{C}$.

\section{RAYTRACING MODEL}

Complementarily to the thermodynamic receiver model, a raytracing analysis has been performed with the in-house tool STRAL [26] to accomplish several related tasks, the most crucial being:

- Predict required heliostats during the solar tests for desired intercept power

- Optimize receiver geometry and positioning
- Analyse and set design parameters for the secondary concentrator

- Check adequateness of heliostat selection algorithm at different power levels and times

- Provide realistic boundary conditions for the thermodynamic receiver model (v.s.) and CFD model [13]

The DLR Solar Tower in Jülich [7] is located at 50.913 ${ }^{\circ}$ latitude north (longitude 6.388 ). Its heliostat field including optical properties based on deflectometric data and the tower geometry are available as libraries for STRAL; the raytracing can thus be processed for an arbitrary flat shaped aperture at any position on the tower and tilt angles.

The model is extended via an additional DLL, in which the receiver geometry implemented for further ray-processing. The module is written in $\mathrm{C}++$ and based on existing codes enabling multi-core processing. The implemented geometry of the receiver and secondary (Figure 9) is represented by a total of nine planes. The raytracing algorithm is mainly based on identification of hit secondary surface, line-plane intersection, reflection and extinction. It considers the free arrangement of each plane in space to freely vary the length of receiver cavity $l_{\text {rec }}$, the diameter of the absorber $D_{a b s}$, the diameter of the window $D_{\text {win }}$, the aperture size of the secondary $D_{a p}$, and its opening angle $\alpha$.

The cylindrical inner insulation surface is not explicitly modelled, since all rays penetrating the receiver either hit the absorber or the insulation. The lumped model accounts for the energy fraction on the absorber (see above), and the CFD model [13] requires the irradiation distribution at the window, further processing all rays itself.

Six planes are arranged to form the secondary. In a latter phase of the project, it was decided to apply a conical shape, but the available hexagonal model can still represent the conical shape as good approximation.

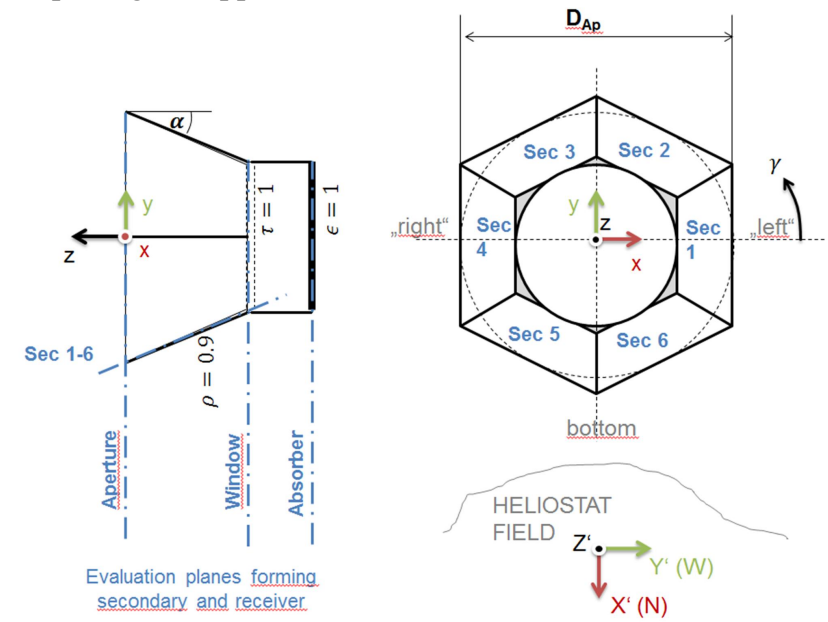

Figure 9: Implemented geometry of the receiver for ray processing in STRAL [26] 


\section{Studies and results of raytracing mode}

The raytracing model allowed for optimizing receiver and secondary geometry in order to reach the most homogeneous radiation flux densities and highest possible incident fraction on the absorber, together with a reasonable reduction of required heliostats. The premise was keeping the secondary shape simple, in tune with the pragmatic receiver design; both are not optimized in terms of optical and thermal efficiency for the demonstration.

Among the considered parameter range and for discrete cases regarding date, time and DNI (consistent to the test campaign schedule), an aperture diameter of $0.6 \mathrm{~m}$ and an opening angle of $25^{\circ}$ yielded a reasonable compromise between reduction of required heliostats, size of the secondary and improvement of homogeneity and incident fraction on absorber. A tilt angle of $16^{\circ}$ was found to provide the best symmetry and thus lowest deviations between maximum and minimum irradiation flux on the absorber.

Deviations from the receiver geometry as derived with the thermodynamic model - such as modifying the cavity length or absorber diameter - were not found to provide crucial improvements. In addition, CFD analyses [13] showed the importance of the radiation distribution from the solar field on temperature distributions and overall thermal performance of the receiver, likely making small changes to geometry insignificant. Figure 10 shows the irradiation flux distribution in the receiver cavity for the final secondary and receiver geometry, and close to nominal power of $57 \mathrm{~kW}(63 \mathrm{~kW}$ on window). Shown are two curves along the horizontal and vertical cut. Mean values for incident flux on the window, absorber, and insulation are additionally marked. This calculation is performed with worst case assumptions regarding time (end of October, 13:00) and DNI $\left(650 \mathrm{~W} / \mathrm{m}^{2}\right)$, with 583 heliostats focused on the target. Active heliostats, as shown in Figure 13, are chosen via a simple cone-plane intersection algorithm. For this case, the concentration factor of the secondary is 1.68 , and the share incident on the absorber $\delta_{C S P}$ is 0.62 .

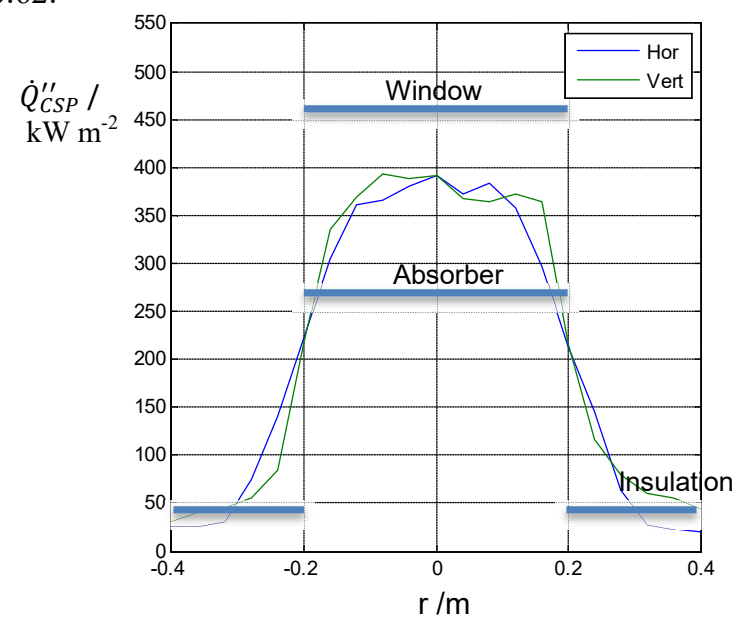

Figure 10: Irradiation flux distribution in the cavity for fina geometry and nominal operation (worst case time / DNI scenario).
Additionally, the model provided the input to determine appropriate cooling methods of the secondary, such as absorbed heat and possible hot spots. It was found that absorbed heat flux is $25 \mathrm{~kW} / \mathrm{m}^{2}$ for the final secondary geometry at nominal irradiation, and hot spots due to multi-reflection only occur for larger sizes of the secondary. Convective cooling with air jets has been considered as thermodynamically feasible, but water cooling was assessed as the operationally more robust option. The latter is realized by a second steel sheet forming a circumferential gap, and the cooling water is routed by a tack welded copper coil between the metallic layers.

\section{PLANT COMISSIONING}

Pilot plant setup was finished in 2015 and a total of four on-sun test runs were performed. The most representative test is shown in Figure 11: The plant was operated with air and water up to $600 \mathrm{ml} / \mathrm{min}$, with maximum incident power on secondary aperture of $49.5 \mathrm{~kW}$, obtained during this day with 322 active heliostats (see also Figure 13). This corresponds to $65 \%$ of nominal power with $1 \mathrm{l} / \mathrm{min}$ acid as described above (compare to Figure 8).

The incident power is measured discretely at aspired operational conditions with a moving bar system [27], when long term steady state values of absorber front and outlet temperatures were approached. Due to temporal variations in irradiation conditions, short term fluctuations in temperature are always present over the long term steady values, as shown in Figure 11.

The trends of three exemplary temperature measurements are shown: 1) Maximum temperature from a total of 8 thermocouples located at $1 / 3$ of the absorber depth. 2) An infrared camera system located in the field at $85 \mathrm{~m}$ distance additionally measures the absorber front temperature via an optical filter for $2.4 \mu \mathrm{m}$ wavelength. 3) Another sun-blind broadband filter is used to measure the window temperature. Window and absorber temperature are measured alternatingly due to the required filter change. However, the maximum temperature measured in the absorber by means of thermocouples was found to closely represent the infrared measurements of the absorber front temperatures (compare Figure 11).

\section{Preliminary validation of receiver model}

The operational point with highest mass flow and incident power in Figure $11(600 \mathrm{ml} / \mathrm{min}$ and $49.5 \mathrm{~kW}$ on aperture $)$ is reproduced with the raytracing model, shown in Figure 12. The irradiation flux measured with the moving bar system (left) and the predicted flux map (right) correspond well, both quantitatively and qualitatively. The focal point during the test is shifted downwards by $10-20 \mathrm{~cm}$. In the marked region of interest (secondary aperture), the shapes of the flux distribution are in good agreement with deviations below $10 \mathrm{~kW} / \mathrm{m}^{2}$ or $7 \%$. 


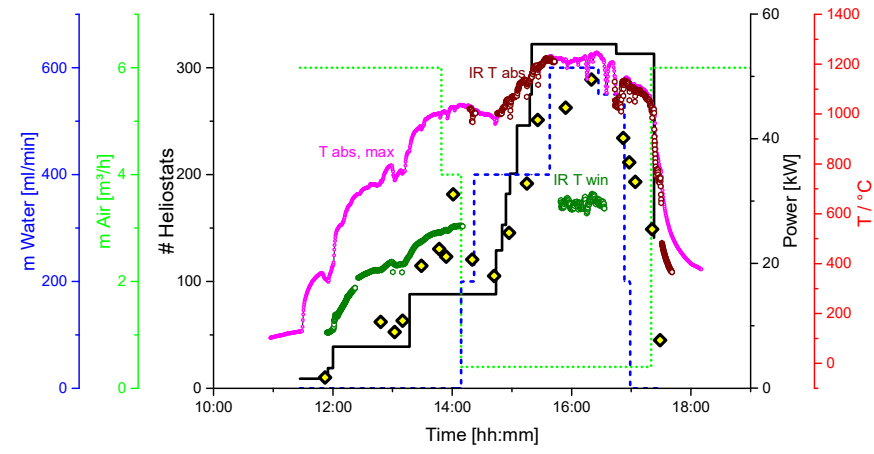

Figure 11: Initial operation in October 2015; water operation with up to $600 \mathrm{ml} / \mathrm{min}$ and incident power up to $50 \mathrm{~kW}$ on the aperture.

Active heliostats are shown in Figure 13 for the same operational point. Note that some heliostats within the active region were not available during the test, e.g. due to simultaneous calibration works. The algorithm to choose the active heliostats during the test is based on a simple cone-plane intersection with given tilt angle of the receiver $\left(16^{\circ}\right)$. The "viewing angle" of an observer viewing through the receiver window is increased (up to approximately $50^{\circ}$ ) with the power requirement.

Appropriate operational points of the presented solar test run were eventually re-simulated with the thermodynamic model. The most crucial parameters of interest, impacting performance and functional integrity of the receiver, are the temperatures of the window, of the absorber front, and of the fluid at the absorber outlet.
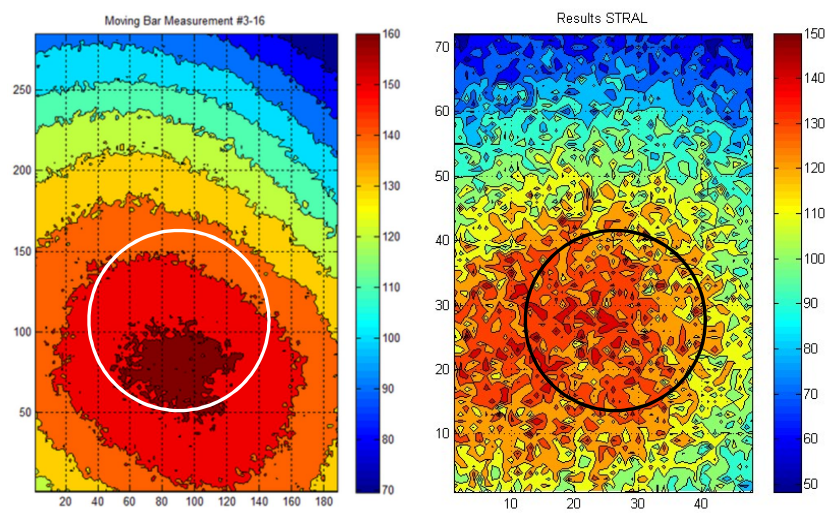

Figure 12: Irradiation flux density measured with the moving bar system (left) and recalculated with STRAL (right) for the considered operational point. Region height $x$ width $(1.3 \times 0.865 \mathrm{~m})$ corresponds to $285 \times 189$ pixels in the measurement and a resolution of $72 \times 48$ in the calculation.

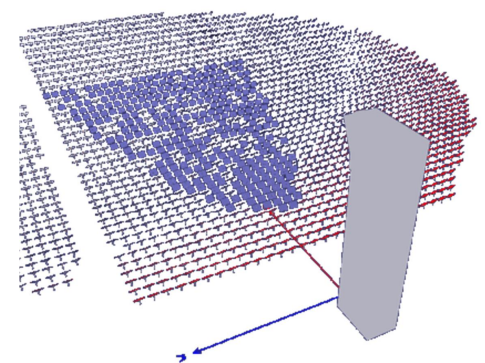

Figure 13: Active heliostat field during the considered operational point; based on a cone-plane intersection algorithm / availability.

A correction factor to consider the optical efficiency of the secondary - the ratio between incident power on the aperture and the incident power on the window - was derived by a comparison of measured and calculated incident power while keeping the fluid outlet temperature equal. Mass flows lower than $400 \mathrm{ml} / 1$ were excluded assuming that the measured fluid outlet temperature is considerably impacted during heating up or cooling down of the receiver by radiation in the manifold, since fluid and material will not be in thermal equilibrium. The correction factor was found to vary between 0.8 and 0.65 , reasonably decreasing with increasing intercept power (broader active mirror field). A preliminary constant factor of 0.75 yielded a good agreement of the outlet temperatures, with maximum deviations of $\pm 60{ }^{\circ} \mathrm{C}$ for operational points with 150 300 active heliostats. This is shown in Figure 14 (triangles), as well as the resulting coincidence of the absorber front temperature (circles) between model (abscissae) and experiment (ordinate). Against design intention as described above, the absorber front temperature is underestimated by the model; apparently with systematic deviation between approximately $+200{ }^{\circ} \mathrm{C}$ (at lower temperatures) and $+100{ }^{\circ} \mathrm{C}$ (at higher temperatures), except for one operational point. A visual inspection of the absorber during assembly found several pores to be clogged, which is one possible explanation of higher maximum front temperatures than expected. Deriving the optical efficiency for each operational point with the raytracing model might yield more precise results and partially address the systematic deviation.

An almost linear characteristic between the maximum measured window temperature and maximum measured absorber temperature was identified as shown in Figure 15. Other dependencies on mass and irradiation flux are small, and the trend is hardly impacted by the fact that all non-steady operational points are shown as well. The window temperature is thus dominated by radiation and re-radiation (i.e. by the absorber front temperature) and the inner heat convection shows a small effect, at least with the experimentally reached mass flows

Two calculated characteristics for different water mass flows $(0.3$ and $1.2 \mathrm{l} / \mathrm{min})$ show a similar trend. With lower mass flows, respective absorber front temperatures are reached at lower irradiation (and thus lower absorber CSP power indicated by the circle size), yielding a slightly lower window 
temperature. The modelling assumptions to estimate the window temperature are thus confirmed twofold. First, radiation is the dominant mechanism and the resulting relationship between absorber and window temperature might be useful for further simplified design approaches. Second, the model over-predicts the window temperature as intended for conservative design. However, the model shows a stronger dependency on the absorber front temperature than measured (larger slope). Possible reasons will be investigated in a deeper validation when operational data with nominal irradiation and mass flow up to $11 / \mathrm{min}$ of sulfuric acid is available. Internal convective cooling will impact the gradient of the relationship. Also note that the absorption of the CSP irradiation is currently set to $5 \%$, being almost double of the determined value (see Table 1).

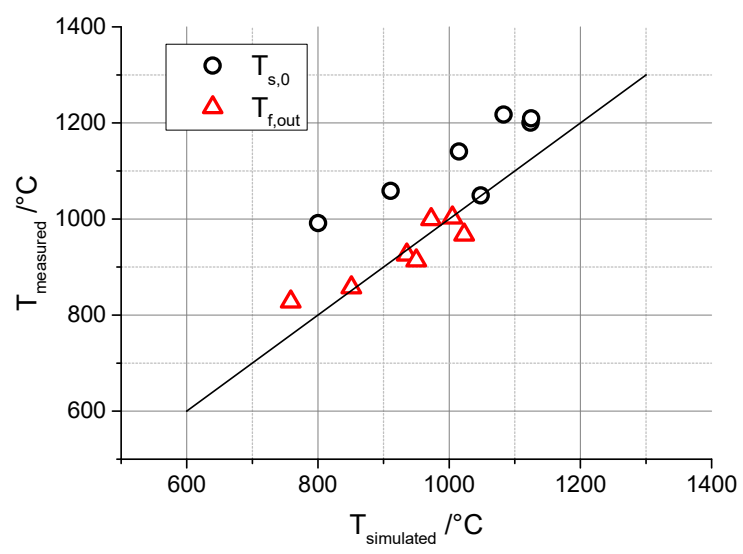

Figure 14: Direct comparison of measured against simulated values: Fluid outlet temperature $T_{\text {fout }}$ (considering a constant factor for optical secondary efficiency of $\mathbf{0 . 7 5}$ ) and absorber front temperature $T_{s, 0}$. The line indicates exact agreement.

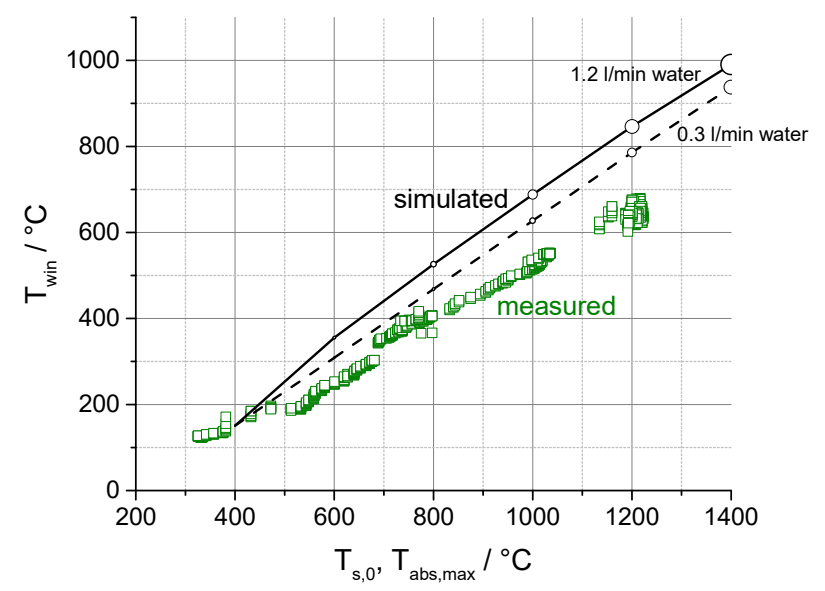

Figure 15: Window temperate $T_{\text {win }}$ measured during solar operation (all available data) and predicted by the model for two mass flow cases as function of the absorber front temperature $T_{s, 0}$ or $T_{a b s, \max }$, respectively.

\section{CONCLUSION AND OUTLOOK}

The SOL2HY2 volumetric receiver for solar superheating of evaporated sulfuric acid to above $1000{ }^{\circ} \mathrm{C}$ is a core component of the demonstration plant built at the DLR Solar Tower in Jülich. A lumped thermodynamic model was set up to analyze different configurations and derive operational parameters of the receiver. Crucial physics - heat transfer in the absorber, radiation heat exchange, temperature dependent effective optical properties of the window - are implemented mathematically robust. All variables can be fixed or kept free depending on the subject of investigation. A first comparison with experimental data obtained during initial on-sun operation shows the thermodynamic lumped model to be a viable tool for design and operational predictions. The main measures of interest - impacting functionality and efficiency - are the component temperatures (window, absorber) and the fluid outlet temperature.

Additionally, a raytracing model has been established to provide the irradiation boundaries and support the design of a secondary concentrator. Available libraries to model heliostat field and tower forecasted operational irradiation on the receiver aperture with less than $7 \%$ deviation in the region of interest in the first sample. For the simple conical geometry, an aperture diameter of $0.6 \mathrm{~m}$ and an opening angle of $50^{\circ}$ yielded the best compromise between reduction of required heliostats, optical efficiency, and size of the secondary.

With a provisional approach to account for the efficiency of the secondary with a constant value of 0.75 as derived by tuning of the fluid outlet temperature, window and absorber temperature as predicted with the thermodynamic model coincide in a very satisfactory manner with the measurements, taking into account the conservative design approach. An absorber front temperature of $1200{ }^{\circ} \mathrm{C}$ was reached at maximum power and mass flow of $50 \mathrm{KW}$ (at secondary aperture) and $600 \mathrm{ml} / \mathrm{min}$ water; $100{ }^{\circ} \mathrm{C}$ higher than predicted with the model. The respective deviation decreases with increasing power levels. Despite higher absorber front temperatures - possibly due to clogged pores in the ceramic foam - the operational temperature of the window has still been overestimated, which was intended for a conservative design. The window temperature has a direct relationship with the absorber front temperature. At an absorber temperature of 1200 ${ }^{\circ} \mathrm{C}$, the measured glass temperature was below $700{ }^{\circ} \mathrm{C} ; 100{ }^{\circ} \mathrm{C}$ lower than predicted. The model shows potential for improvement to better reflect the relationship between glass and absorber temperature. A deeper validation will be performed with pending operational data at higher mass flows and irradiation flux, including the comparison of side wall temperatures to account for less significant loss mechanisms. Aspired nominal mass flow is $11 / \mathrm{min}$ of sulfuric acid, superheated to $1000{ }^{\circ} \mathrm{C}$ by $57 \mathrm{~kW}$ incident power at the window (estimated $76 \mathrm{~kW}$ at secondary aperture).

The SOL2HY2 receiver is kept robust with a flat ceramic porous absorber in an insulated cylindrical casing closed by a 
quartz glass window. Main purpose is to prove feasibility of high temperature superheating in a closed volumetric receiver and adiabatic decomposition reaction, demonstrated at relevant scale in a real operational environment.

More complex receiver and secondary geometries, optimized for optical and thermal efficiency, will be considered for future projects. The presented models as well as more sophisticated dynamic models as reported in [6] will be adapted accordingly, e.g. considering different boundaries and viewing factors of a cavity design. A coupling to overall process models as reported in [28] will allow analysing yearly yields and costs of a directly irradiated, solar HyS-process.

One target is to perform a direct benchmark of directly irradiated and indirectly heated and solar concepts. Modelling approaches for the latter case are reported by CORGNALE AND SUMMERS [29] and CONNOLLY et al. [30].

\section{ACKNOWLEDGMENTS}

This work has been funded by the European Fuel Cells and Hydrogen Joint Undertaking (FCH-JU) project SOL2HY2 (contract no. 325320).

The authors would like to acknowledge the intense efforts by Mariarosaria Ferrara (ENEA), Stefan Schmitz (DLR), Marcel Pißner (DLR), Björn Freitag (DLR), and Dr. Moises Romero (DLR) regarding plant design and commissioning.

The presented mathematical solution for the energy balance in the absorber has been developed by Peter Schwartzbötzl (DLR). Amadeus Rong (DLR) supported the compilation process of the raytracing model with $S T R A L$.

\section{References}

[1] L. E. Brecher, S. Spewock, and C. J. Warde, "The Westinghouse Sulfur Cycle for the thermochemical decomposition of water," International Journal of Hydrogen Energy, vol. 2, no. 1, pp. 7-15, 1977.

[2] W. A. Summers, J. L. Steimke, D. T. Hobbs, H. R. Colon-Mercado, and M. B. Gorensek, "Development of a Sulfur Dioxide Depolarized Electrolyzer for Hydrogen Production Using the Hybrid Sulfur Thermochemical Process," in Fourth International Topical Meeting on High Temperature Reactor Technology, pp. 527-529.

[3] Kolb G. J, Diver R. B, "Screening Analysis of Solar Thermochemical Hydrogen Concepts: SANDIA Report," Albuquerque, NM, 2008.

[4] A. Noglik, M. Roeb, C. Sattler, and R. Pitz-Paal, "Experimental study on sulfur trioxide decomposition in a volumetric solar receiver-reactor," Int. J. Energy Res, vol. 33, no. 9, pp. 799-812, 2009.

[5] D. Thomey, L. de Oliveira, J.-P. Säck, M. Roeb, and C. Sattler, "Development and test of a solar reactor for decomposition of sulphuric acid in thermochemical hydrogen production," International Journal of Hydrogen Energy, vol. 37, no. 21, pp. 16615-16622, 2012.
[6] N. Bayer Botero, D. Thomey, A. Guerra Niehoff, M. Roeb, C. Sattler, and R. Pitz-Paal, "Modelling and scaling analysis of a solar reactor for sulphuric acid cracking in a hybrid sulphur cycle process for thermochemical hydrogen production: accepted," International Journal of Hydrogen Energy, 2016.

[7] G. Koll, P. Schwarzbözl, K. Hennecke, T. Hartz, M. Schmitz, and B. Hoffschmidt, Eds, The Solar Tower Jülich - A Research and Demonstration Plant for Central Receiver Systems, 2009.

[8] General Atomics, "Decomposition of sulfuric acid using solar thermal energy (GA-A17573)," 1985.

[9] General Atomics, "High-pressure catalytic metal reactor in a simulated solar central receiver, GA-A18285," 1986.

[10] M. B. Gorensek and W. A. Summers, "Hybrid sulfur flowsheets using PEM electrolysis and a bayonet decomposition reactor," International Journal of Hydrogen Energy, vol. 34, no. 9, pp. 4097-4114, 2009.

[11] A. Giaconia, S. Sau, C. Felici, P. Tarquini, G. Karagiannakis, C. Pagkoura, C. Agrafiotis, A. G. Konstandopoulos, D. Thomey, L. de Oliveira, M. Roeb, and C. Sattler, "Hydrogen production via sulfur-based thermochemical cycles: Part 2: Performance evaluation of $\mathrm{Fe} 2 \mathrm{O} 3$-based catalysts for the sulfuric acid decomposition step," International Journal of Hydrogen Energy, vol. 36, no. 11, pp. 6496-6509, 2011.

[12] F-Chart Software, EES: Engineering Equation Solver.

[13] J. L. Lapp, A. Guerra-Niehoff, H.-P. Streber, D. Thomey, M. Roeb, and C. Sattler, "Modeling of a Solar Receiver for Superheating Sulfuric Acid," pp. V002T14A001.

[14] H.-P. Streber, A. Guerra Niehoff, D. Thomey, M. Roeb, and C. Sattler, "Application fo CAE Tools for design and scaling of a solar reactor and receiver for acid splitting for the HyS process at pilot plant scale," in International CAE Conference 2015

[15] S. Möller, S. Friedmann, M. Walter, and J. ten Dam, "SOLREF: Development of an Advanced Solar HighTemperature Reformer," in ASME 2006 International Solar Energy Conference, pp. 269-276.

[16] N. Piatkowski and A. Steinfeld, "Solar gasification of carbonaceous waste feedstocks in a packed-bed reactorDynamic modeling and experimental validation," AIChE $J$, vol. 57, no. 12, pp. 3522-3533, 2011.

[17] A. Veeraragavan, A. Lenert, B. Yilbas, S. Al-Dini, and E. N. Wang, "Analytical model for the design of volumetric solar flow receivers," International Journal of Heat and Mass Transfer, vol. 55, no. 4, pp. 556-564, 2012.

[18] E. J. Sheu and A. F. Ghoniem, "Receiver reactor concept and model development for a solar steam redox reformer," Solar Energy, vol. 125, pp. 339-359, 2016.

[19] H. D. Baehr and K. Stephan, Heat and mass-transfer, 2nd ed. Berlin, New York: Springer, 2006.

[20] VDI Gesellschaft, Ed, VDI-Wärmeatlas: [Berechnungsunterlagen für Druckverlust, Wärme- und 
Stoffübertragung], 10th ed. Berlin, Heidelberg [u.a.]: Springer, 2006.

[21] J. R. Howell, R. Siegel, and M. P. Mengüç, Thermal radiation heat transfer.

[22] T. Fend, B. Hoffschmidt, R. Pitz-Paal, O. Reutter, and P. Rietbrock, "Porous materials as open volumetric solar receivers: Experimental determination of thermophysical and heat transfer properties," Energy, vol. 29, no. 5-6, pp. 823-833, 2004.

[23] B. Hoffschmidt, "Vergleichende Bewertung verschiedener Konzepte Volumetrischer Strahlungsempfänger,” RWTH Aachen, 1996.

[24] Adrien Schwane, "Modellierung und Simulation eines neuartigen Luft-Solarreceivers mit Cavity," Thesis, RWTH Aachen, 2014.

[25] M. Röger, M. Pfänder, and R. Buck, "Multiple Air-Jet Window Cooling for High-Temperature Pressurized Volumetric Receivers: Testing, Evaluation, and Modeling," J. Sol. Energy Eng, vol. 128, no. 3, p. 265, 2006.

[26] B. Belhomme, R. Pitz-Paal, P. Schwarzbözl, and S. Ulmer, "A New Fast Ray Tracing Tool for HighPrecision Simulation of Heliostat Fields," J. Sol. Energy Eng, vol. 131, no. 3, p. 31002, 2009.

[27] Gereon Feckler, Benjamin Wittenberg, Stefan Schmitz, Christian Willsch, Marcel Pißner, Martin Thelen, Andreas Schierkes, Felix Göhring, Thomas Fend, "High density solar radiation flux measurement system for research solar tower," Solar Energy, vol. brief note submitted, currently in review.

[28] A. Guerra Niehoff, N. Bayer Botero, A. Acharya, D. Thomey, M. Roeb, C. Sattler, and R. Pitz-Paal, "Process modelling and heat management of the solar hybrid sulfur cycle," International Journal of Hydrogen Energy, vol. 40, no. 13, pp. 4461-4473, 2015.

[29] C. Corgnale and W. A. Summers, "Solar hydrogen production by the Hybrid Sulfur process," International Journal of Hydrogen Energy, vol. 36, no. 18, pp. 11604 11619, 2011

[30] S. M. Connolly, E. Zabolotny, D. F. McLaughlin, and E. J. Lahoda, "Design of a composite sulfuric acid decomposition reactor, concentrator, and preheater for hydrogen generation processes," International Journal of Hydrogen Energy, vol. 34, no. 9, pp. 4074-4087, 2009. 